\title{
A reduced complexity scheme for carrier frequency synchronization in uplink 802.16e OFDMA
}

\author{
Javier González-Bayón *, Angel Fernández-Herrero and Carlos Carreras
}

\begin{abstract}
Synchronization of the carrier frequency offset (CFO) in the uplink of OFDMA systems is a complex task because each user presents a different CFO. It is performed in two stages: estimation and compensation. Although a few existing schemes already achieve good performance in terms of bit error rate (BER), all of them perform huge amounts of operations, specially during estimation, thus leading to very costly, if not impractical, hardware implementations. In addition, if estimation is performed in the time domain, a preamble is required. Since the IEEE 802.16e standard for OFDMA has no preamble at the beginning of the uplink frame, optional midambles must be used at the cost of reducing the system throughput. This article proposes a combined CFO estimation and compensation technique that uses the pilot subcarriers of the $802.16 \mathrm{e}$ frame. It achieves similar BER performance than previous approaches without any throughput penalty, while reducing the number of required computations by several orders of magnitude. This reduction is the result of a simpler estimation algorithm and two techniques that improve its accuracy: an iterative process integrated with the compensation stage and the delayed correction of the phase shifts.
\end{abstract}

Keywords: Carrier frequency offset (CFO), Orthogonal frequency division multiple access (OFDMA), Synchronization, 802.16e, Mobile WiMAX

\section{Introduction}

The increasing demand for multimedia communications with variable data rates and different quality of service requirements has recently led to a strong interest in OFDMA. This transmission technique has been chosen for mobile wireless metropolitan area network as in 802.16e-2005 (mobile WiMAX) [1]. In OFDMA, subcarriers are grouped into sets, each of which is assigned to a different user. Block, interleaved, random or clusteredtiled assignment schemes can be used for this purpose. Unfortunately, OFDMA is particularly sensitive to the different carrier frequency offsets (CFOs) caused by oscillator mismatches between the users and the base station (BS) and/or by Doppler shifts. All the users transmitting in the uplink signal have to be synchronized with the BS in order to prevent inter-symbol interference, intercarrier

*Correspondence: javier@die.upm.es

Dpto. de Ingeniería Electrónica, E.T.S.I. Telecomunicación, Universidad Politécnica de Madrid, 28040 Madrid, Spain interference (ICI), multiple access interference (MAI), signal attenuation, and a constant increment in the phase of the subcarriers. ICI is caused by the leakage or interference between a user's own subcarriers, whereas MAI is caused by the power leakage from other users' subcarriers.

Frequency synchronization in OFDMA is based on a three-step procedure. In the first step, each user performs CFO estimation and correction in the downlink. This operation reduces the CFO and can easily be accomplished using techniques applied in OFDM systems [2-6]. The obtained CFO estimates are used as synchronization references in the subsequent uplink transmission. Due to estimation errors in the first step and/or oscillator instabilities and/or Doppler effects, uplink signals arriving at the BS may be affected by some residual CFOs, which not only introduce ICI and MAI, but also cause a phase rotation in the subcarriers, thus producing incorrect data decisions after a few OFDM symbols. Hence, the second necessary step is to perform CFO estimation in the uplink. The third step is the compensation of the CFO at the receiver, which also requires different techniques than

\section{Springer}

(c) 2012 González-Bayón et al: licensee Springer. This is an Open Access article distributed under the terms of the Creative Commons Attribution License (http://creativecommons.org/licenses/by/2.0), which permits unrestricted use, distribution, and reproduction in any medium, provided the original work is properly cited. 
those used in OFDM systems. This article focuses on the second and third steps.

Several approaches have been presented for interleaved or block subcarrier assignments [7-11], but just a few methods are available for the random and clustered cases. Iterative CFO estimation schemes for the random case are found in [12-17]. They can also be used for other permutation cases.

In [12], a complete OFDMA detection scheme is proposed, but the complexity of the calculations makes impossible its practical hardware implementation.

In [13-16], a preamble amended at the beginning of the uplink frame is used to perform CFO estimation in the time domain. However, there is no preamble at the beginning of the uplink frame in the 802.16e standard. This standard supports optional repeating short preambles, called midambles, one out of 4,8 , or 16 OFDM symbols [1]. Although midambles can support the implementation of these estimation methods, they also decrease the throughput of the system. In addition, huge amounts of operations are still required by these schemes.

In [17], pilot subcarriers are used to estimate and correct the CFOs by performing an iterative process. This process is composed of an exhaustive search step and a frequency correction step that uses the ICI matrix defined in [17]. Again, an exhaustive search is a very expensive approach in terms of the required computations. Although a limited sweep is proposed to alleviate this problem, the tradeoff between accuracy and complexity remains uncharacterized.

In $[18,19]$, compensation methods for the different CFOs are proposed. However, the estimation part is not included in these studies. The method in [19] reduces the number of computations required in [18] by taking advantage of the physical frame of the 802.16e standard, where subcarriers are grouped into tiles.

In this article, a novel scheme to estimate and compensate for the CFOs that reduces the number of computations with no performance degradation with respect to previous CFO synchronization schemes is proposed. The computational complexity of the algorithms is a key issue when determining the number of hardware resources needed for portable, battery-powered systems. In this sense, the proposed scheme has been designed with the goal in mind of using a relatively simple estimation method and still achieving good bit error rate (BER) results. This has been possible through two specific optimization techniques: (1) a feedback from the compensation stage that allows the iterative elimination of the ICI and MAI during estimation, and (2) the delayed correction of the phase shift so it is performed after the iterative process, thus increasing the accuracy of the CFO estimates.
In addition, the proposed synchronization method is suitable for communication standards that do not include a preamble as part of the uplink specification, like mobile WiMAX, since it uses the pilot subcarriers available in the data frame. Therefore, there are no throughput losses in the communication system.

The performance of this method depends on the position and number of pilot subcarriers. Such performance is evaluated for the 802.16e uplink tile assignment called uplink partially used subcarrier (UL PUSC). BER results are shown to determine the performance of the synchronization algorithm compared to other proposals. Also, the number of computations of the proposed method is compared to other frequency synchronization schemes.

The article is structured as follows. In "The WiMAX frame" and "The OFDMA signal" sections, the WiMAX permutation modes and the OFDMA signal are introduced, respectively. Other existing CFO synchronization methods are presented in "Previous OFDMA synchronization schemes" section. "Proposed synchronization scheme" section describes the proposed synchronization scheme. Simulation results are given in "Simulation results" section, and computation complexities are analyzed in "Computational complexity" section. Finally, "Conclusions" section concludes the article.

\section{The WiMAX frame}

The IEEE 802.16e standard defines a physical layer that can use 128, 512, 1024, or 2048 subcarriers, which are modulated with BPSK, QPSK, or 16-QAM constellations. A number of boosted subcarriers are allocated for pilot signals and a number of the highest and lowest frequency subcarriers are null. A subchannel is a logical collection of subcarriers that is assigned to a specific user. The number and exact distribution of the subcarriers that constitute a subchannel depends on the subcarrier permutation mode.

There are two types of subcarrier permutations for subchannelization: diversity and contiguous. The diversity permutation includes UL PUSC, UL O-PUSC (optional uplink partially used subcarrier) and also several downlink modes.

UL PUSC splits the time-frequency grid into tiles. This permutation mode can be observed in Figure 1. Six tiles are grouped to form a slot. The slot comprises 48 data subcarriers and 24 pilot subcarriers in 3 OFDM symbols. The minimum frequency-time resource unit of subchannelization is one slot. In a similar way, UL O-PUSC is composed of tiles of eight data subcarriers and one pilot subcarrier in three OFDM symbols.

The contiguous permutation (also called UL AMC) groups a block of nine contiguous subcarriers in a symbol, with eight of them assigned to data and one assigned to a pilot. 


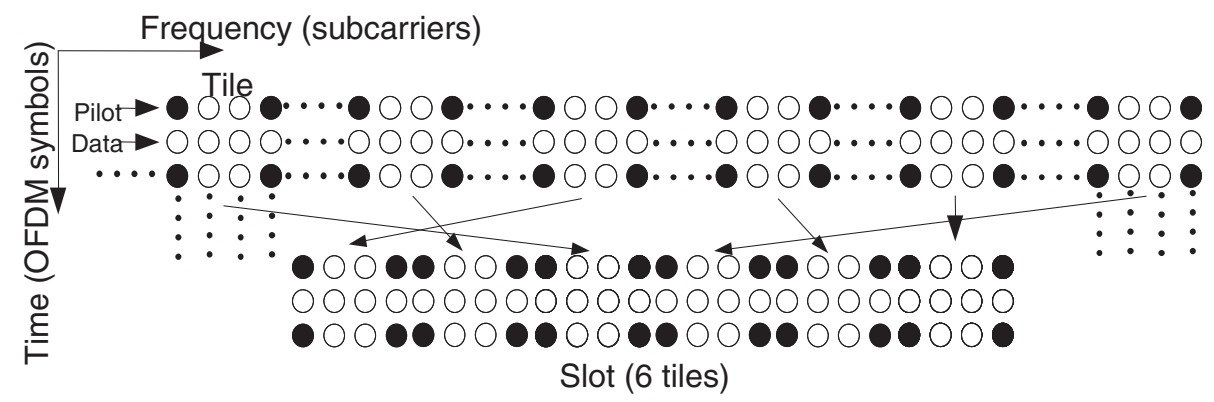

Figure 1 Structure of tiles for UL PUSC.

The pilot subcarriers of the three permutation modes are BPSK modulated. In UL O-PUSC and UL AMC, they are transmitted at a boosted power level of $4 / 3$.

\section{The OFDMA signal}

An OFDMA system with $D$ simultaneously active users and $N$ subcarriers is considered. The inverse discrete Fourier transform of an OFDM symbol of the $i$ th transmitter user can be written as

$$
x_{i}(n)=\sum_{k \in \Gamma_{i}} X_{i}(k) e^{-j \frac{2 \pi k n}{N}}-N_{g} \leq n \leq N-1
$$

where $N_{g}$ is the length of the cyclic prefix $(\mathrm{CP})$ and $X_{i}(k)$, $k \in \Gamma_{i}$ is the value of the transmitted symbol on the $k$ th subcarrier. The set of subcarriers assigned to user $i$ is denoted as $\Gamma_{i}$. These sets satisfy $\cup_{i=1}^{D} \Gamma_{i}=\{0,1, \ldots, N-1\}$ and $\Gamma_{i} \cap \Gamma_{j}=\emptyset$ if $j \neq i$. Vectors and matrices are denoted by bold-face letters in the following equations. Assuming perfect timing synchronization, the discrete-time model of the received signal at the BS, after removal of the $\mathrm{CP}$, can be written in matrix form for an OFDMA symbol as

$$
\boldsymbol{y}=\sum_{i=1}^{D} \boldsymbol{D}\left(\varepsilon_{i}\right) \boldsymbol{P}\left(\boldsymbol{x}_{i}\right) \boldsymbol{h}_{i}+\boldsymbol{w}
$$

where $\boldsymbol{D}\left(\varepsilon_{i}\right)=\operatorname{diag}\left(1, e^{-j 2 \pi \varepsilon_{i} / N}, \ldots, e^{-j 2 \pi(N-1) \varepsilon_{i} / N}\right), \boldsymbol{h}_{i}$ is a vector of length $L$ that encapsulates the channel response for the $i$ th user, $\boldsymbol{y}=\left\{y_{0}, \ldots, y_{N-1}\right\}, \boldsymbol{P}\left(\boldsymbol{x}_{i}\right)$ is an $N \times L$ matrix with entries $\left[\boldsymbol{P}\left(\boldsymbol{x}_{i}\right)\right]_{k, l}=x_{i}(k-l)$ where $0 \leq k \leq N-1$ and $0 \leq l \leq L-1, \boldsymbol{w}$ is a white Gaussian noise with variance $\sigma^{2}$, and $\varepsilon_{i}=\Delta f / f_{\text {sub }}$ is the CFO for the $i$ th user normalized with respect to the subcarrier spacing.

The receiver applies a DFT to signal $y$ to recover the frequency domain symbols. The DFT output at the $k$ th subcarrier can be obtained as

$$
Y_{m}(k)=\sum_{i=1}^{D}\left(\sum_{u \in \Gamma_{i}} X_{i, m}(u) H_{i, m}(u) G\left(u, k, \varepsilon_{i}\right)\right) \Delta_{i, m}+W(k)
$$

where $\Delta_{i, m}=e^{j 2 \pi \frac{m N_{s}+N_{g}}{N} \varepsilon_{i}}, \boldsymbol{X}_{i}, \boldsymbol{H}_{i}$ and $\boldsymbol{W}$ are the DFTs of $\boldsymbol{x}_{i}, \boldsymbol{h}_{i}$ and $\boldsymbol{w}$, respectively, $m$ is the OFDM symbol index, and $N_{s}=N_{g}+N$.

$G\left(u, k, \varepsilon_{i}\right)$ is the leakage across subcarriers due to the frequency offset [18]. It can be formulated as

$$
G\left(u, k, \varepsilon_{i}\right)=e^{j \pi\left(u-k+\varepsilon_{i}\right) \frac{N-1}{N} \varepsilon_{i}} \frac{\sin \pi\left(u-k+\varepsilon_{i}\right)}{N \sin \frac{\pi\left(u-k+\varepsilon_{i}\right)}{N}}
$$

Assuming that $k \in \Gamma_{i}$, the received signal in the $k$ th subcarrier of the $i$ th user can be written as

$$
\begin{aligned}
Y_{m}(k)= & \underbrace{X_{i, m}(k)}_{\text {Desired value }} H_{i, m}(k) \underbrace{e^{j 2 \pi \frac{m N_{s}+N_{g}}{N} \varepsilon_{i}}}_{\text {Phase shift: } \Delta_{i, m}} G\left(k, k, \varepsilon_{i}\right) \\
& +\underbrace{\Delta_{i, m} \sum_{\substack{u \in \Gamma_{i} \\
u \neq k}}\left(X_{i, m}(u) H_{i, m}(u) G\left(u, k, \varepsilon_{i}\right)\right)}_{\text {MAI }} \\
& +\underbrace{\sum_{u \in \Gamma_{j}}^{D} \sum_{j, m}(u) H_{j, m}(u) G\left(u, k, \varepsilon_{j}\right) \Delta_{j, m}}_{\substack{j=1 \\
j \neq i}}+W(k)
\end{aligned}
$$

where the first term is the desired signal $(u=k)$ with amplitude reduction and phase distortion, while the second and third terms represent the ICI and the total MAI, respectively. The system model considered so far is independent of the subcarrier allocation method.

\section{Previous OFDMA synchronization schemes}

Different frequency synchronization schemes for random tile assignment in the uplink of OFDMA systems can be found in the literature. Some of them just focus on the estimation stage or the compensation stage. Comparisons of the proposed scheme with these previous approaches are presented in "Simulation results" and "Computational complexity" sections.

A CFO estimation scheme for generalized subcarrier allocation in OFDMA systems has been proposed in [14], in which CFO estimation is performed by assuming that there is a preamble at the beginning of the 
frame. The method maximizes the log-likelihood function for unknown parameters $\varepsilon_{i}$ using the preamble. Unfortunately, solving the resulting equation requires an exhaustive search over the parameter space, which is prohibitively complex for practical implementation. An approach, called alternating-projection frequency estimator (APFE), that replaces the search over a multidimensional domain by a sequence of mono-dimensional searches is proposed to reduce such complexity. However, despite some improvement, the requirements of computational resources still remain extremely high.

To circumvent the previous problem, Morelli et al. [15] propose an iterative scheme, in which a space-alternating generalized expectation-maximization (SAGE) algorithm [20] is first used to extract the signal of each user, say $r_{i}$. Each $r_{i}$ is then used to estimate the corresponding $\varepsilon_{i}$ by following an expectation-conditional maximization approach. This leads to a procedure consisting of cycles and iterations with two main stages called the E-step and the M-step. The number of cycles corresponds to the number of users: $D$. In every iteration, CFO estimates are updated, and the process continues until no significant changes are observed.

A preamble is required to perform the SAGE algorithm but, as previously mentioned, there are standards like $802.16 \mathrm{e}$ where no preamble is included at the beginning of the uplink frame. However, it is possible to use optional midambles defined in mobile WiMAX at the cost of decreasing the throughput of the system. In addition, the SAGE algorithm still performs a large amount of computations, thus requiring a costly hardware implementation.

In addition, there still remains the problem of the compensation. The total ICI (and MAI) matrix can be computed and a maximum likelihood (ML) correction can be performed for CFO cancellation as in [18]. However, this requires obtaining the inverse of the ICI matrix which, again, implies a large number of computations due to its $N \times N$ size. Unfortunately, this complexity is unacceptable for implementation purposes.

There is another option to correct the effect of the CFO, which takes into account the tile structure of the 802.16e frame [19]. By using this method, an iterative compensation scheme that uses a small ICI matrix is performed. This scheme is called decorrelation successive cancellation (DC-SC) and it is explained in the next section. In any case, the methods in $[18,19]$ only approach the compensation problem and do not include an estimation stage.

In [17], a synchronization method that estimates and compensates the CFOs is presented. It is called pilotaided tile structure-based frequency synchronization system (PATS). It proposes a physical frame composed of frequency domain tiles of five subcarriers with one pilot subcarrier in the middle. The synchronization is performed by an iterative joint CFO estimation and compensation scheme. The CFO of an individual user is determined from a one-dimensional search where the MAI from other users is treated as noise.

CFO estimation in PATS is performed by applying a nonlinear least squares algorithm where an exhaustive search needs to be performed. The iterative joint CFO estimation and compensation is aimed at reducing the complexity of the multi-dimensional search to a onedimensional search. CFO estimates are then exploited to compensate for the ICI and MAI in the next iteration, and the corrected signal is used to estimate the residual CFOs. CFO compensation is performed by using the method in [18], where the effect of the CFO is represented as an interference matrix. This compensation scheme requires the inversion of the large interference matrix, which is very demanding computationally as the size of the matrix is determined by the number of subcarriers $(N)$ in one OFDM symbol.

PATS is performed in the frequency domain and uses the pilot subcarriers of the tiles, thus avoiding the use of midambles. It is presented as a low complexity scheme because it has lower complexity than the APFE estimation method in [14]. However, as shown in "Simulation results" section, PATS still has an extremely high complexity that prevents any practical implementation.

Taking all of this into account, new proposals are needed. The goal is to design a complete frequency synchronization scheme (i.e., including estimation and compensation) that, not only achieves good BER performance, but also has reduced resource requirements and no throughput losses. These new proposals should be evaluated with uplink $802.16 \mathrm{e}$ frames, since this is currently the main standard using OFDMA.

\section{Proposed synchronization scheme}

This study proposes a frequency synchronization scheme that, like PATS, implements an iterative approach during estimation supported by a feedback loop from the compensation stage to the estimation stage. However, not only the estimation and compensation stages are different from the ones in PATS, but also the motivation to include the feedback is different. While PATS uses the feedback to reduce the complexity of the estimation stage, in the proposed scheme the feedback is used to increase the otherwise insufficient accuracy of the estimation stage.

The estimation stage of the proposed scheme is partially based on a technique for CFO tracking for OFDM systems. However, this technique alone cannot estimate medium or high CFO values accurately. This problem is solved by means of an iterative process supported by the feedback from the compensation stage. A second technique, based on using non-consecutive symbols to obtain 
the phase rotation, is also applied to increase the accuracy of the estimation and reduce the cost of the fixed-point hardware implementation.

The compensation scheme used in the proposal is the method in [19]. This method has lower complexity than the one in [18]. It also helps to reduce the ICI and MAI noise during estimation through the proposed iterative scheme, thus improving the accuracy of the estimation stage, as explained in the following sections.

\section{Estimation technique}

At the receiver, after applying FFTs on two time-domain blocks $\left(y_{m}\right.$ and $\left.y_{m+M}\right)$, the frequency domain $k$ th subcarrier from Equation (5) becomes

$$
\begin{aligned}
Y_{m}(k)= & X_{i, m}(k) H_{i, m}(k) \underbrace{e^{j 2 \pi} \frac{m N_{s}+N_{g}}{N} \varepsilon_{i}}_{\Delta_{i, m}} G\left(k, k, \varepsilon_{i}\right) \\
& +W_{\mathrm{ICI}}+W_{\mathrm{MAI}}+W(k) \\
Y_{m+M}(k)= & X_{i, m+M}(k) H_{i, m+M}(k) \underbrace{e^{j 2 \pi \frac{(m+M) N_{s}+N_{g}}{N}} \varepsilon_{i}}_{\Delta_{i, m+M}} G\left(k, k, \varepsilon_{i}\right) \\
& +W_{\mathrm{ICI}}^{\prime}+W_{\mathrm{MAI}}^{\prime}+W^{\prime}(k)
\end{aligned}
$$

As it can be observed, the difference between both expressions is in the factors $\Delta_{i, m}$ and $\Delta_{i, m+M}$ that multiply the data signal, assuming that the CFO and the channel remain nearly constant over the $M$ OFDM symbols. Hence, it is possible to use a frequency-domain CFO estimation technique as in [3], which uses the pilot subcarriers available in the data frame. The adaptation of this scheme to an OFDMA scenario is done by performing the algorithm $D$ times, once for each user and its specific pilot subcarriers.

In [3], CFO estimates are obtained using $M$ consecutive OFDM symbols. The post-FFT correlation of Equations (6) and (7) at the $p$ th pilot subcarrier of the $i$ th user is

$$
C_{i}(p)=\sum_{l=1}^{M-1} Y_{m+l}^{*}(p) Y_{m+l-1}(p)
$$

where $p \in \Gamma_{p i}$ and $\Gamma_{p i}$ are the set of pilot subcarriers assigned to user $i\left(\Gamma_{p i} \subset \Gamma_{i}\right)$. This scheme is proposed in [3] for $M=2$. CFO values can then be estimated as

$$
\varepsilon_{i}=\frac{N}{4 \pi N_{s}}\left[\arg \sum_{p} C_{i}(p)\right]
$$

The idea of using the phase rotations of several consecutive OFDM symbols is taken one step further in the proposed approach. The phase rotation between two consecutive OFDM symbols due to the CFO is

$$
\Phi_{2}=2 \pi \frac{N_{s}}{N} \varepsilon=2 \pi\left(1+\frac{N_{g}}{N}\right) \varepsilon
$$

while the phase rotation between the first and third OFDM symbols in a sequence of consecutive OFDM symbols becomes

$$
\Phi_{3}=2 \pi \frac{2 N_{s}}{N} \varepsilon=4 \pi\left(1+\frac{N_{g}}{N}\right) \varepsilon
$$

Since the rotation in (11) is twice the rotation in (10) for the same noise, more accurate CFO estimations can be performed with (11) than with (10). Even though using the first and third OFDM symbols reduces the CFO estimation range by half, this should not be a problem as long as CFOs are small.

Thus, the CFO can be estimated by comparing the phases of the pilot subcarriers of two OFDM symbols separated by $M$ OFDM symbols. Extending the expression in [3]

$$
\begin{aligned}
C_{i}(p) & =Y_{m}^{*}(p) Y_{m+M}(p) \\
& =\underbrace{e^{j \pi \frac{M N_{s}}{N} \varepsilon_{i}}}_{\Delta_{i, M}} \alpha^{2}\left(\varepsilon_{i}\right)|H(p)|^{2} \beta^{2} \sigma_{a}^{2}+w
\end{aligned}
$$

where $\sigma_{a}^{2}$ is the power of the transmitted symbols, $\beta^{2}$ is the boosted power factor of the pilot subcarriers, and $\alpha$ is the attenuation caused by the CFO as described in (4). Notice that the phase factor $\Delta_{i, M}$ allows that the CFO value can be obtained as

$$
\varepsilon_{i}=\frac{N}{2 \pi M N_{s}}\left[\arg \sum_{p} C_{i}(p)\right]
$$

This proposed method, that has also been applied in [6], is called data-aided phase incremental technique (DAPIT), and it assumes that $\varepsilon_{i}$ and the channel are nearly static over $M$ consecutive OFDM symbols.

One important issue of the UL PUSC frame is that there are no continual pilot subcarriers, since not every OFDM symbol has pilot subcarriers. As it can be observed in Figure 1, there are two simple ways of using the pilot subcarriers in the UL PUSC mode to perform the necessary correlation prior to CFO estimation. It can either be calculated using the pilot subcarriers of two consecutive OFDM symbols, or the pilot subcarriers of one OFDM symbol and the third consecutive OFDM symbol. Therefore, Equations (12) and (13) can be applied directly to both cases, whereas (8) and (9) are not valid for the second one, since they only perform the correlation over consecutive OFDM symbols.

Another important advantage of using DA-PIT is that it can also be used with the other permutation schemes of the uplink 802.16e, as UL O-PUSC or UL AMC, which have different pilot and tile structures. 


\section{Reduced complexity compensation method}

As it was previously mentioned, the compensation stage is performed with the DC-SC method [19]. In this scheme, an interference matrix is built for each group of $K$ subcarriers that form a tile or cluster. For example, the interference matrix for UL PUSC has a $4 \times 4$ size, e.g., $K=4$. By using this small matrix, the total correction of the ICI and MAI noise cannot be performed as it is proposed in [18]. Instead, ICI interference is removed in each tile by multiplying the subcarriers of the tile by the inverse of this small ICI matrix. This stage is called decorrelation. A second procedure, called successive cancellation, is used to remove the MAI of the current tile on the other tiles. The $K \times K$ matrix $\Pi_{i}$ is the interference matrix, whose entries are $\Pi_{i}(u, k)=G\left(u, k, \varepsilon_{i}\right)$, where $G$ is given in (4). This matrix is invertible for $-1<\varepsilon_{i}<1$, as it is shown in [19].

In the DC-SC scheme, the clusters are first sorted in descending order according to their average power. Then, starting from the cluster $c$ with the largest power which belongs to $i$ th user, decorrelation is applied to every subcarrier in the cluster. This is represented as

$$
\hat{\boldsymbol{y}}_{c}=\boldsymbol{\Pi}_{i}^{-1} \boldsymbol{y}_{c}
$$

where $\boldsymbol{y}_{c}=\left[Y_{i}(k) \ldots Y_{i}(k+K)\right], c \in \Gamma_{i}^{c}$, and $\Gamma_{i}^{c}$ span the subcarriers from $k$ to $k+K$ (i.e., $\Gamma_{i}^{c}=k, \ldots, k+K$ ). This decorrelation corrects the ICI of the tile and, after that, the channel is cancelled on the $\hat{\boldsymbol{y}}_{c}$ signal and the demapping is performed. Therefore, in ideal conditions, the transmitted data subcarriers of the tile are obtained. These data subcarriers are used in the next step that is called successive cancellation.

After obtaining the partially corrected subcarriers in the current cluster, the MAI from the neighboring clusters is reconstructed and canceled using the knowledge of the frequency offset value. ICI from another cluster of the same $i$ th user is treated as MAI in this procedure. The data subcarriers and the estimation of the channel (assuming that it was obtained from previous OFDM symbols) are used to accomplish that. Therefore, the MAI can be reconstructed without noise or interference if the data decisions are correct. After removal of the MAI from the $c$ th cluster, the $u$ th $\left(u \notin \Gamma_{i}^{c}\right)$ subcarrier value becomes [19]

$$
\hat{Y}(u)=Y(u)-(\hat{\boldsymbol{X}} \hat{\boldsymbol{H}})_{c}^{T} \boldsymbol{g}_{c, u}
$$

where $\boldsymbol{g}_{c, u}=\left[G\left(k, u, \varepsilon_{i}\right), G\left(k+1, u, \varepsilon_{i}\right) \ldots G\left(k+K, u, \varepsilon_{i}\right)\right]$ and $(\hat{\boldsymbol{X}} \hat{\boldsymbol{H}})_{c}^{T}$ is the transmitted signal for this tile, obtained after the demapping is performed with the channel values. The successive cancellation stage needs correct data decisions on the demapper to perform the cancelation accurately.
In the proposed scheme (see below), the DC-SC method uses (14) and (15) in $Y_{m}$ and $Y_{m+M}$ to cancel the ICI and MAI noise in order to improve the accuracy of the estimation stage.

\section{Proposed integrated scheme}

Among the previously proposed schemes, the combined application of the SAGE estimation [15] and the DC-SC compensation [19] probably constitutes the most balanced solution when considering cost as well as performance (see Figure 2a). When SAGE performs two iterations during estimation, this scheme achieves good BER results with less computational resources than other existing schemes. However, the amount of computations is still very large, leading to very costly hardware implementations, and SAGE also requires the use of midambles in mobile WiMAX, resulting in reduced system throughput.

A pilot-based scheme that requires less resources than SAGE + DC-SC can be obtained by combining the DAPIT estimation technique with DC-SC (see Figure 2b). However, as shown in the next section, if medium or high CFO values must be estimated in high SNR conditions, DA-PIT alone is not sufficient to achieve acceptable BER results in OFDMA systems due to the high ICI and MAI noise.

An integrated CFO estimation and compensation (IEC) scheme is proposed here to solve this problem (see Figure 2c). It implements an iterative scheme through a feedback from the DC-SC compensation stage that increases the accuracy of the DA-PIT estimation stage,

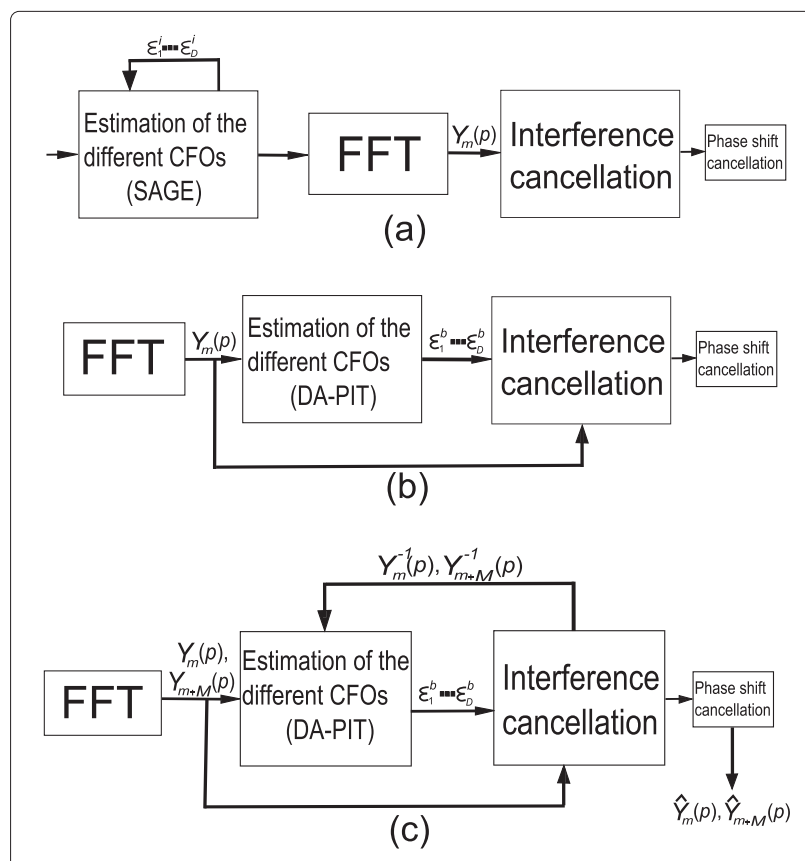

Figure 2 Block diagrams of different synchronization schemes. (a) SAGE + DC-SC, (b) DA-PIT + DC-SC, (c) IEC. 
as it allows the iterative cancellation of the ICI and MAI noise [Equations (6) and (7)] during the estimation process. The estimation accuracy is further improved by considering non-consecutive symbols when obtaining the phase shift used by DA-PIT to estimate the CFO.

The proposed approach avoids the internal iterations for estimating the different CFOs that occur in SAGE. In addition, the phase changes produced by the CFOs are not corrected. In particular, the ICI and MAI are removed from (6) and (7), but the phase factors $\Delta_{m}$ and $\Delta_{m+M}$ produced by the CFO still remain. These factors are not corrected as part of the proposed IEC scheme because of two main reasons.

First, the amount of operations is reduced as a consequence of using the DA-PIT estimator which is based on these phase shift factors [Equations (12) and (13)]. And second, each new CFO estimation is not iteratively added to the previous one, as it occurs in PATS where a similar iterative scheme is also proposed [17]. By not correcting this phase shift, the new CFO estimation substitutes the previous one, thus maintaining the range of its numeric value, instead of decreasing it as it happens in [17]. In consequence, the digital representation of the CFO estimates in a hardware implementation (obtained after a quantization process) can be maintained across iterations, thus requiring fewer bits than in other schemes to obtain the same accuracy. This favors the use of reduced size fixedpoint operators and, therefore, improves the area (cost), power consumption, and performance of the hardware implementation.

When the iterative estimation process converges and the last ICI and MAI compensation is performed, the phase shift $\Delta$ is finally corrected.

In summary, the iterative algorithm can be described in terms of the following steps.

1. Iterative part:

(a) Estimation of residual CFOs according to (12) and (13) for $D$ different users $\rightarrow \varepsilon_{i}$

(b) Updating of $\boldsymbol{\Pi}_{i}(u, k)$ using (4) with $\varepsilon_{i}$

(c) Compensation of ICI and MAI according to (14) and (15) using $\Pi_{i}(u, k)$

(d) Return to (a) using the OFDM symbols corrected in (c) until convergence is reached

2. Phase shift cancellation

It is important to realize that the compensation in step (c) is always applied to the original OFDM symbols, not to the symbols corrected in previous iterations.

The simplest criterion to stop the iterative process is to consider a predefined fixed number of iterations. As in other previous iterative schemes, simulations show that after two iterations improvements are negligible, so IEC always performs only two iterations.

\section{Simulation results}

Simulations have been performed for four different users ( $D=4$ ) that communicate to the BS using the UL PUSC 802.16e frame mode and are modulated with a QPSK constellation. The FFT has $N=512$ points and there are 200 pilot subcarriers. The CFOs introduced in the simulations are in the range $\{-0.1,+0.1\}$. According to the standard [1], the maximum allowed residual normalized CFO after downlink synchronization is 0.02 (2\%). However, larger CFOs have been considered to account for possible oscillator instabilities and Doppler effects. The length of the tile is $K=4$ since the UL PUSC frame is considered.

A Rayleigh channel consisting of two paths with an average attenuation of $-3 \mathrm{~dB}$ is used in the simulations. For simplicity, it is assumed that this channel and also the timing synchronization are perfectly estimated.

The MSE of the CFO estimators and the BER performance of the complete schemes are computed by averaging the offset error values and bit error values throughout 15,000 simulations. BER results are shown for one OFDM symbol.

Figure 3 shows MSE results of the CFO estimation in UL PUSC mode comparing the SAGE algorithm with one and two iterations, the DA-PIT method, and the estimation of the proposed IEC scheme. It can be observed that both methods using some feedback (IEC and SAGE with two iterations) have much better accuracy in their estimations. In fact, both methods obtain similar performance for all SNR values. It can also be observed that there is a flat MSE response for high SNR values. This is caused by the residual ICI and MAI noise that is not corrected during the estimation process.

Figure 4 shows the BER curves for the UL PUSC permutation mode. The schemes being compared are SAGE + DC-SC (with one and two SAGE iterations), DAPIT + DC-SC, and the proposed IEC. The SAGE + DC-SC synchronization is performed using a midamble with the structure of an OFDM symbol (512 samples) of the UL PUSC 802.16e frame [1]. The PATS scheme has been discarded in this comparison because its high complexity prevents any practical hardware implementation (see next section). Only the $24-40 \mathrm{~dB}$ SNR range is shown in the plot because this is where the differences between schemes show up and also where acceptable BER values are obtained. The curves tend to overlap for smaller SNRs, thus providing little information.

It is observed that the BER of the two non-iterative methods, SAGE(1ite) + DC-SC and DA-PIT + DC-SC, decreases at high SNRs compared to the offset free case. This is because at high SNRs the ICI and MAI 


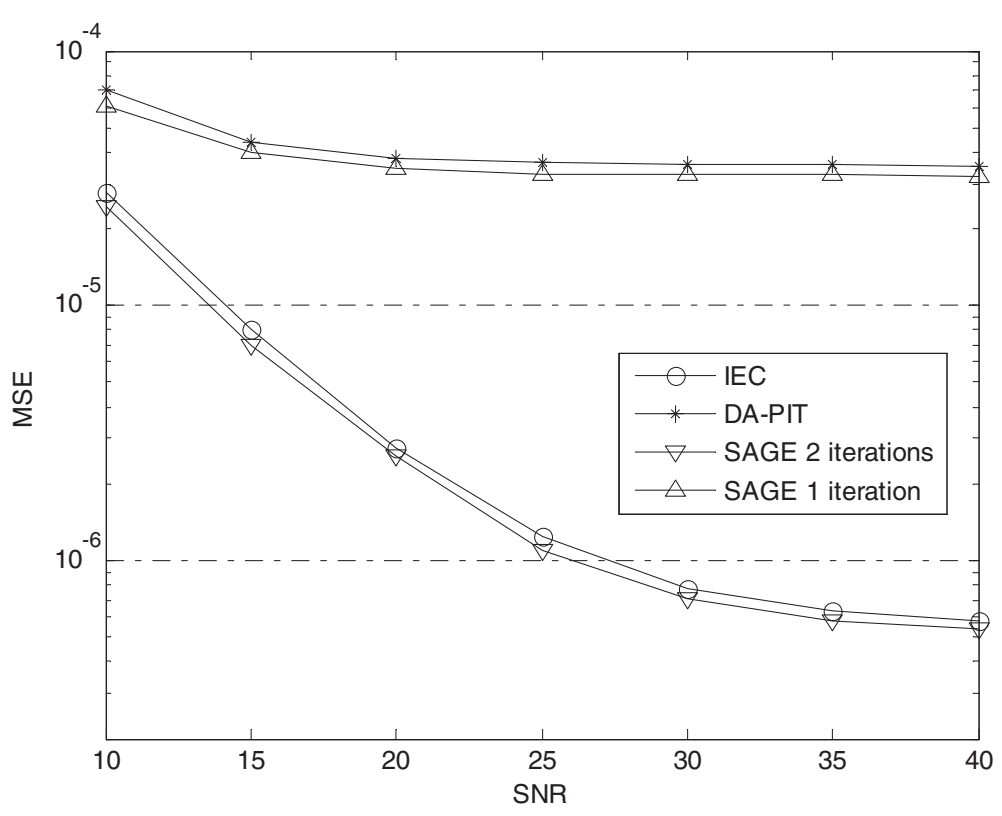

Figure 3 MSE results for UL PUSC.

residual noise is the main source of noise that degrades the system performance. However, the other two methods, SAGE(2ite) + DC-SC and IEC, have good BER performance even at high SNRs because the ICI and MAI noise is reduced during the iterative estimation process. It is remarkable that both methods achieve very similar performance since their complexities are very different, with IEC being much simpler (see next section). In addition,
IEC does not add extra data in the transmission frame since it does not use the optional midamble.

\section{Computational complexity}

There still remains an important issue that needs to be considered for implementation purposes: the computational complexity of the different schemes. Computations are quantified in terms of the number of multiplications,

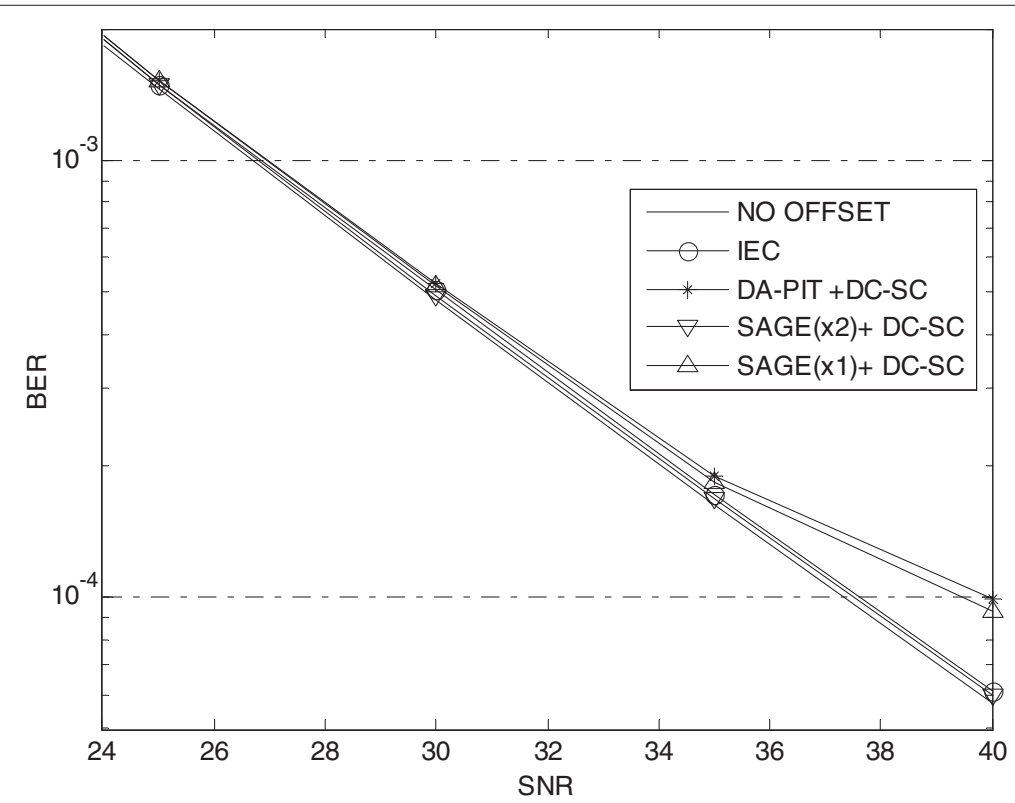

Figure 4 BER results for UL PUSC. 
since they are more costly than additions in fixed-point architectures.

According to [17], the number of multiplications in PATS is $N_{b}\left(2 N_{r} N Z K K_{p}+N^{3}\right)$, where $K_{p}$ is the number of pilot subcarriers per OFDM symbol, $N_{r}$ is the number of points for the exhaustive search and $Z$ the number of tiles per user.

Since PATS uses the compensation in [18], but the DC-SC method [19] has similar performance but less complexity, we also consider here an alternative iterative scheme called ES+DC-SC that combines the exhaustive search estimation of PATs called ES with the DC-SC compensation. The goal is to assess the impact of the compensation approach. Since the computational cost of ES is $2 N_{r} N Z K K_{p}$ and DC-SC performs $N(N+K) / 2+K^{2}$ multiplications, the total cost of ES+DC-SC is $N_{b}\left(2 N_{r} N Z K K_{p}+\right.$ $\left.N(N+K) / 2+K^{2}\right)$.

According to [15], the number of multiplications to obtain a CFO estimation using SAGE after $N_{b}$ iterations is $N_{b}\left(2 N K L N_{r}\right)$, where $L$ is the number of taps of the channel. Therefore, the cost of SAGE + DC-SC is $N_{b}\left(2 N K L N_{r}\right)+N(N+K) / 2+K^{2}$.

Finally, the proposed IEC scheme requires $N$ multiplications per iteration in the DA-PIT estimation stage, $N(N+$ $K) / 2+K^{2}$ multiplications in the DC-SC compensation stage, and $N$ additional multiplications in the final phase shift compensation. Thus, considering $N_{b}$ iterations, it performs $N_{b}\left(N(N+K) / 2+K^{2}\right)+N$ multiplications. This expression also applies to the DA-PIT + DC-SC scheme considering that $N_{b}=1$.

For example, Figure 5 shows the computational complexity of these schemes (i.e., multiplications in a logarithmic scale) when considering a UL PUSC mode with $N=512$, a tile of length $K=4, Z=20$ tiles, a channel of $L=16$ taps, a search over $N_{r}=1000$ points as stated in [17], one $\left(N_{b}=1\right)$ and two $\left(N_{b}=2\right)$ iterations, and assuming that the different users occupy all the available bandwidth. It should be remembered that only the

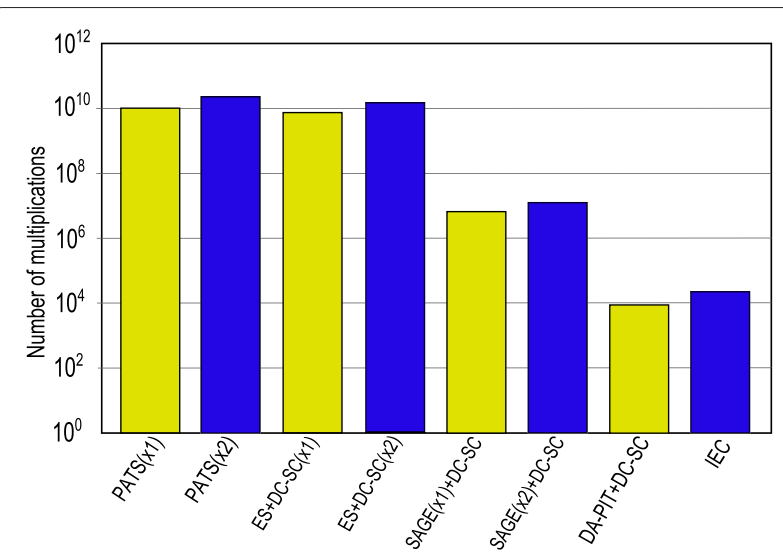

Figure 5 Number of multiplications per synchronization scheme. schemes performing two iterations achieve good BER performance. Their complexities are shown with dark bars. As for the non-iterative $\left(N_{b}=1\right)$ schemes including DAPIT + DC-SC (light bars), they help to assess the impact of the iterations in each case.

It is observed that neither using different compensation methods (PATS versus ES + DC-SC) nor performing two iterations instead of one causes dramatic differences in the complexities of the schemes. It is the actual schemes that have very different complexities. PATS and ES + DCSC perform around $10^{10}$ multiplications, thus justifying that they were not considered in the previous section due to their huge computation requirements. At the other end, the proposed IEC only performs around $10^{4}$ multiplications while still achieving good BER performance, thus becoming the best option when considering cost and performance.

\section{Conclusions}

A new frequency offset estimation scheme for the uplink of an 802.16e OFDMA system has been presented. This complete synchronization scheme, named IEC, has been designed with the goal of matching the good BER performance found in other existing schemes, but also reducing their huge computational complexities so that low-cost hardware implementations are feasible. Three specific techniques have been used in the design: a low-cost OFDM estimation method adapted to OFDMA, and two optimizing techniques aimed at improving its accuracy. First, using an iterative process integrated with the compensation stage, and second, not correcting the phase shifts as part of the iterative procedure.

It has been shown that the IEC method obtains similar performance to the SAGE + DC-SC combination, a state-of-the-art synchronization scheme, even for relatively high CFO values and without the throughput losses caused by the use of midambles required by the SAGE estimation.

Furthermore, the IEC proposal reduces by two and five orders of magnitude the number of multiplications required by the SAGE + DC-SC and PATS schemes, respectively. In addition, IEC can also be used for other tile permutation modes of the 802.16e standard, like UL O-PUSC or UL AMC, with no added complexity, thus becoming a feasible cost-effective frequency synchronization solution for this standard.

Competing interests

The authors declare that they have no competing interests.

\section{Acknowledgements}

This study was supported in part by the Spanish Ministry of Science and Innovation under contract TEC2009-14219-C03-02. 


\section{References}

1. Amended to 802.16e: IEEE Standard for Local and Metropolitan Area Networks Part 16, Air Interface for Fixed Broadband Wireless Access Systems. Amendment for Physical and Medium Access Control Layers for Combined Fixed and Mobile Operation in Licensed Bands and Corrigendum 1, IEEE 802.16-2005, December 2005

2. P Moose, A technique for orthogonal frequency division multiplexing frequency offset correction. IEEE Trans. Commun. 42, 2908-2914 (1994)

3. M Speth, S Fechtel, G Fock, H Meyr, Optimum receiver design for OFDM-based broadband transmission. Part II: a case study. IEEE Trans. Commun. 49(4), 571-578 (2001)

4. J González-Bayón, C Carreras, O Edfors, A multi-standard frequency offset synchronization scheme for $802.11 \mathrm{n}, 802.16 \mathrm{~d}$, LTE and DVB-T/H systems. J. Comput. Syst. Netw. Commun. 2010, 1-9 (2010)

5. T Schmidl, D Cox, Robust frequency and timing synchronization for OFDM. IEEE Trans. Commun. 45, 1613-1621 (1997)

6. J González-Bayón, A Fernández-Herrero, C Carreras, Improved schemes for tracking residual freequency offset in DVB-T systems. IEEE Trans. Consumer Electron. 56(2), 415-422 (2010)

7. S Barbarossa, M Pompili, G Giannakis, Channel independent synchronization of orthogonal frequency division multiple access systems. IEEE J. Sel. Areas Commun. 20(2), 296-306 (2002)

8. J Van de Beek, P Borjesson, M Boucheret, D Landstrom, J Arenas, O Odling, $M$ Wahlqvist, S Wilson, A time and frequency synchronization scheme for multiuser OFDMA uplink. IEEE J. Sel. Areas Commun. 17(11), 1900-1914 (1999)

9. Z Cao, U Tureli, YD Yao, in Proc. IEEE ICC, Efficient structure-based carrier frequency offset estimation for interleaved OFDMA uplink. vol. 5, May 2003, pp. 3361-3365

10. D Huang, KB Letaief, An interference-cancellation scheme for carrier frequency offset correction in OFDMA systems. IEEE Trans. Commun. 53(7), 1155-1165 (2005)

11. J Choi, C Lee, HW Jung, YH Lee, Carrier frequency offset compensation for uplink of OFDM-FDMA systems. IEEE Commun. Lett. 4(12), 414-416 (2000)

12. M-O Pun, M Morelli, C-C Jay Kuo, Iterative detection and frequency synchronization for OFDMA uplink transmissions. IEEE Trans. Wirel. Commun. 6(2) (2007)

13. X-N Zeng, A Ghrayeb, Joint CFO and channel estimation for OFDMA uplink: an application of the variable projection method. IEEE Trans. Wirel. Commun. 8(5), 2306-2311 (2009)

14. M Pun, M Morelli, C Kuo, Maximum-likelihood synchronization and channel estimation for OFDMA uplink transmissions. IEEE Trans. Commun. 54(4), 726-736 (2006)

15. M Morelli, C-C Jay Kuo, M-O Pun, Synchronization techniques for orthogonal frequency division multiple access (OFDMA): a tutorial review. Proc. IEEE. 95(7), 1394-1427 (2007)

16. HC Nguyen, E de Carvalho, R Prasad, in Proc. of the IEEE 19th Int. Symp. on Personal, Indoor and Mobile Radio Communications, A generalized carrier frequency offset estimator for uplink, OFDMA. 1-5 September 2008

17. P Sun, L Zhang, Low complexity pilot aided frequency synchronization for OFDMA uplink transmission. IEEE Trans. Wirel. Commun. 8(7), 3758-3769 (2009)

18. Z Cao, U Tureli, YD Yao, P Honan, in Proc. of the IEEE Global Telecommunications Conference, Frequency synchronization for generalized OFDMA uplink. vol. 2, November 2004, pp. 1071-1075

19. T Yücek, H Arslan, Carrier frequency offset compensation with successive cancellation in uplink OFDMA systems. IEEE Trans. Wirel. Commun. 6(10), 3546-3551 (2007)

20. J Fessler, A Hero, Space-alternating generalized expectation-maximization algorithm. IEEE Trans. Signal Process. 42(10), 2664-2677 (1994)

doi:10.1186/1687-6180-2012-218

Cite this article as: González-Bayón et al:: A reduced complexity scheme for carrier frequency synchronization in uplink 802.16e OFDMA. EURASIP Journal on Advances in Signal Processing 2012 2012:218. 